\title{
Modification of interval arithmetic in modeling uncertainty of boundary conditions in boundary value problems
}

\author{
M. Kapturczak ${ }^{\mathrm{a}}$, E. Zieniuk ${ }^{\mathrm{a}}$ and K. Szerszeń $^{\mathrm{a}}$ \\ anstitute of Informatics, University of Bialystok, Biatystok, Poland \\ Email: ezieniuk@ii.uwb.edu.pl
}

\begin{abstract}
Nowadays, the finite element method (FEM) is the most known and the most commonly used method for solving boundary value problems defined exactly (without uncertainty). This method is based on the division of the domain into finite elements. These elements are connected to each other and produces a mesh. The second method, more recent, but also often used, is the boundary element method (BEM). This method is based on the division of the boundary only on the so-called boundary elements. This solution significantly reduces the amount of data required to solve the problem. Therefore, the amount of computer resources, needed to obtain a solution, is also significantly reduced. However, both FEM and BEM require a large number of points to define the shape of the boundary. It was the main reason to look for new methods. This is how the parametric integral equations system (PIES) appeared.

In this paper, the application of interval arithmetic for modeling uncertainly defined boundary conditions (in solving boundary value problems) is presented. In the literature, for solving such problems, different modifications of mentioned FEM and BEM methods appeared. Many different ways of modeling uncertainty have been used. However, the most important problem is to reduce the number of calculations. This is the main reason to choose interval arithmetic. Direct application of classical or directed interval arithmetic, into interval modifications of FEM and BEM methods, leads to significant overestimation. Therefore, it was decided to propose interval modification of the parametric integral equations system (PIES) method.

Recently, the PIES method has been thoroughly tested on examples of boundary problems, where input data were defined without uncertainty. During tests, the number of input data (necessary to define the problem) was always smaller (comparing with well-known methods). This also allows reducing the number of equations in the obtained system of equations. This gives a significant advantage (over other methods) in modeling uncertainty of input data.

The application of the interval PIES method resulted in a significant reduction of overestimation. However, obtained solutions (by direct application of interval arithmetic known from literature) are not exactly in line with expectations. Therefore, the modification of directed interval arithmetic has been proposed. It has been applied for calculations in the interval PIES method. The proposed method has been tested on examples of boundary value problems (modeled by Laplace's equation) with uncertainly defined boundary conditions. The uncertainty of boundary conditions has been defined as interval constant boundary conditions as well as interval linear function. Obtained solutions have been compared with the solutions of exactly defined (without uncertainty) PIES.
\end{abstract}

Keywords: Uncertainty, interval arithmetic, boundary value problems, boundary conditions 


\section{INTRODUCTION}

In the literature, the most recently used methods (for modeling and solving boundary value problems) are the finite element method (FEM) (Spiridonov et al., 2019) and boundary element method (BEM) (Kapor et al., 2011).The modification of such methods, to consider the uncertainty of input data using interval numbers, appeared (Sofia et al., 2016; Piasecka-Belkhayat, 2011). However, both FEM and BEM methods need a large number of input data. It is troublesome even with the boundary value problems defined without uncertainty. It was the main reason to develop the parametric integral equations system method (PIES) (Zieniuk et al., 2009, 2015). In this method, the shape of the boundary has been directly included in the mathematical formalism of boundary integral equations (BIE). Therefore, for modeling the shape of the boundary, only control points of the curves (well known from computer graphics) are needed. It significantly reduces the number of input data. It is a very important advantage in modeling uncertainty because allow reducing overestimation.

In this paper, it was decided, to focus on the impact of boundary conditions uncertainty on the solutions obtained by interval PIES. The solutions have been obtained based on examples of Laplace's equation. The uncertainty of boundary conditions, defined as interval constant value as well as interval linear function, has been tested. Obtained interval solutions have been compared with classical PIES solutions (without uncertainty). The correctness of the PIES method, in exactly defined problems (using real numbers), has been previously confirmed by many research publications (Zieniuk et al., 2009, 2015; Zieniuk, Kapturczak, 2018).

The interval PIES method is written as a computer program in C++ language using Visual Studio 2013 compiler. There was a need to modify interval arithmetic, therefore no library was used, all classes and methods were implemented by the authors. Interval arithmetic operations have been implemented as methods in a special class of interval numbers. $\mathrm{C}++$ language has an option of operators overloading. So then the application of interval arithmetic is quite simple, by changing the variables of standard double type to objects of interval numbers class.

\section{INTERVAL NUMBERS}

Interval numbers are one of the most commonly used ways for modeling uncertainty. These numbers are defined by some interval, in which the measured value is certainly included. First, in the literature, the classical interval numbers appeared. The main feature of these numbers is the condition, which ensures that the lower bound is lower than the upper one. The classical interval number can be defined as follow (Moore, 1966):

$$
\boldsymbol{x}=[\underline{x}, \bar{x}]=\{x \in \mathbb{R} \mid \underline{x} \leq x \leq \bar{x}\} .
$$

Same to any set of numbers, there is a need for appropriate arithmetic. The classical interval numbers arithmetic operations count the minimum and maximum values from all of the results on intervals bounds (Moore, 1966):

$$
\boldsymbol{x} \circ \boldsymbol{y}=[\underline{x}, \bar{x}] \circ[\underline{y}, \bar{y}]=[\min (\underline{x} \circ \underline{y}, \underline{x} \circ \bar{y}, \bar{x} \circ \underline{y}, \bar{x} \circ \bar{y}), \max (\underline{x} \circ \underline{y}, \underline{x} \circ \bar{y}, \bar{x} \circ \underline{y}, \bar{x} \circ \bar{y})],
$$

where $\circ \in\{+,-, \cdot, /\}$ and $0 \notin \boldsymbol{y}$. Using properties of arithmetic operations and having regards to interval bounds condition, the above formula can be simplified. Taking addition operation as an example, the minimum value can be obtained by the addition of two lower bounds and the maximum value by two upper bounds. It can be defined as follow:

$$
\boldsymbol{x}+\boldsymbol{y}=[\underline{x}, \bar{x}]+[\underline{y}, \bar{y}]=[\underline{x}+\underline{y}, \bar{x}+\bar{y}] .
$$

Similarly, all other arithmetic operations can be defined.

The interval numbers were mainly used for the estimation of rounding errors, which occur as the result of hardware limitations in numerical computation. However further studies of modeling the uncertainty allow perceiving some disadvantages. For example, the inverse and opposite elements cannot be obtained. Therefore, the solution of the simple equation is significantly overestimated (even more for the system of equations). This is the main reason for the directed interval numbers development. There is no interval bounds condition anymore. The directed intervals can be defined as follows (Markov, 1992):

$$
\boldsymbol{x}=[\underline{x}, \bar{x}]=\{x \in \boldsymbol{x} \mid \underline{x}, \bar{x} \in \mathbb{R}\} .
$$

To determine the directed interval number, two additional variables were applied: sign $(\sigma)$ and direction $(\tau)$ :

$$
\sigma=\left\{\begin{array}{ll}
+ & \text { for } \quad \underline{x}, \bar{x}>0 \\
- & \text { for } \quad \underline{x}, \bar{x}<0
\end{array} \quad, \quad \tau=\left\{\begin{array}{ll}
+ & \text { for } \quad \underline{x}<\bar{x} \\
- & \text { for } \quad \underline{x}>\bar{x}
\end{array} .\right.\right.
$$


The directed interval arithmetic is base on the above-mentioned formula. Obviously, by removing the condition, the problem with obtaining the opposite and inverse elements is not solved. Therefore, it is necessary to define two additional directed interval arithmetic operations (subtraction and division):

$$
\boldsymbol{x} \ominus \boldsymbol{y}=[\underline{x}-\underline{y}, \bar{x}-\bar{y}], \quad \boldsymbol{x} \oslash \boldsymbol{y}=\left\{\begin{array}{lll}
{[\underline{x} / \underline{y}, \bar{x} / \bar{y}]} & \text { for } \quad \boldsymbol{x}>0, \boldsymbol{y}>0 \\
{[\bar{x} / \bar{y}, \underline{x} / \underline{y}]} & \text { for } \quad \boldsymbol{x}<0, \boldsymbol{y}<0 \\
{[\bar{x} / \underline{y}, \underline{x} / \bar{y}]} & \text { for } \quad \boldsymbol{x}>0, \boldsymbol{y}<0 \\
{[\underline{x} / \bar{y}, \bar{x} / \underline{y}]} & \text { for } \quad \boldsymbol{x}<0, \boldsymbol{y}>0 \\
{[\bar{x} / \underline{y}, \underline{x} / \underline{y}]} & \text { for } \quad \boldsymbol{x} \ni 0, \boldsymbol{y}<0 \\
{[\underline{x} / \bar{y}, \bar{x} / \bar{y}]} & \text { for } \quad \boldsymbol{x} \ni 0, \boldsymbol{y}>0
\end{array} .\right.
$$

Presented way of uncertainty modeling, using directed interval arithmetic, significantly reduce overestimation. However, during the research studies of applications of such numbers for modeling and solving uncertainly defined boundary value problems many problems appeared (Zieniuk et al., 2016, 2018). As a result of the research on the problems, the modification of directed interval arithmetic has been proposed. Such modification based on mapping the interval arithmetic operations into positive semi-axis:

$$
\boldsymbol{x} \cdot \boldsymbol{y}=\left\{\begin{array}{ll}
\boldsymbol{x}_{s} \cdot \boldsymbol{y}_{s}-\boldsymbol{x}_{s} \cdot y_{m}-x_{m} \cdot \boldsymbol{y}_{s}+x_{m} \cdot y_{m} & \text { for } \quad \boldsymbol{x} \leq 0, \boldsymbol{y} \leq 0 \\
\boldsymbol{x}_{s} \cdot \boldsymbol{y}-x_{m} \cdot \boldsymbol{y} & \text { for } \quad \boldsymbol{x}>0, \boldsymbol{y} \leq 0 \\
\boldsymbol{x} \cdot \boldsymbol{y}_{s}-\boldsymbol{x} \cdot y_{m} & \text { for } \quad \boldsymbol{x} \leq 0, \boldsymbol{y}>0 \\
\boldsymbol{x} \cdot \boldsymbol{y} & \text { for } \quad \boldsymbol{x}>0, \boldsymbol{y}>0
\end{array},\right.
$$

where for any interval number $\boldsymbol{a}=[\underline{a}, \bar{a}]$ is defined $a_{m}=\left\{\begin{array}{ll}|\bar{a}| & \text { for } \quad \bar{a}>\underline{a} \\ |\underline{a}| & \text { for } \quad \bar{a}<\underline{a}\end{array}\right.$ and $\boldsymbol{a}_{s}=\boldsymbol{a}+a_{m}$. Additionally, $\boldsymbol{a}>0$ means $\underline{a}>0$ and $\bar{a}>0$, when $\boldsymbol{a} \leq 0$ means $\underline{a}<0$ or $\bar{a}<0$. The multiplication $(\cdot)$ is the interval multiplication. The appropriateness of directed interval arithmetic modification has been widely discussed in (Zieniuk et al., 2018) and presented on examples of boundary value problems modeled by Laplace's equation further in this paper.

\section{INTERVAL PARAMETRIC INTEGRAL EQUATIONS SYSTEM}

The FEM and BEM methods, as already mentioned, need discretization. This causes a significant increase in interval input data for uncertainly defined boundary value problems. Therefore, in this paper, the parametric integral equations system (PIES) was proposed. There is no discretization, neither of the domain nor the boundary. The shape of the boundary is directly included in the mathematical formalism of PIES. This allows reducing the number of control points (necessary to define the shape of the boundary). Therefore, the number of equations in the solved system of equations is also reduced.

The boundary value problems, modeled by Laplace's equation with uncertainly defined boundary conditions, were presented. The PIES method, with uncertainly defined boundary conditions, should be defined as follow:

$$
0.5 \boldsymbol{u}_{l}\left(s_{1}\right)=\sum_{j=1}^{n} \int_{\widehat{s}_{j-1}}^{\widehat{s}_{j}}\left\{U_{l j}^{*}\left(s_{1}, s\right) \boldsymbol{p}_{j}(s)-P_{l j}^{*}\left(s_{1}, s\right) \boldsymbol{u}_{j}(s)\right\} J_{j}(s) d s,
$$

where $\widehat{s}_{l-1} \leq s_{1} \leq \widehat{s}_{l}$ and $\widehat{s}_{j-1} \leq s \leq \widehat{s}_{j}$ are exactly defined in the parametric coordinate system and respectively represent the beginnig and the end of the curve segment (that modeling the boundary shape) $\boldsymbol{S}_{m}=\left[S_{m}^{(1)}, S_{m}^{(2)}\right]$, where $m=j, l$.

Function $J_{j}(s)$ is the Jacobian of the curve segment $\boldsymbol{S}_{j}(s)$ (specified by $\mathrm{j}$ index) and is defined as follow:

$$
J_{j}(s)=\left[\left(\frac{\partial S_{j}^{(1)}(s)}{\partial s}\right)^{2}+\left(\frac{\partial S_{j}{ }^{(2)}(s)}{\partial s}\right)^{2}\right]^{0.5},
$$

where $S_{j}{ }^{(1)}(s)$ and $S_{j}{ }^{(2)}(s)$ are the vector components of the curve segment $\boldsymbol{S}_{j}=\left[S_{j}{ }^{(1)}(s), S_{j}{ }^{(2)}(s)\right]^{T}$, which depending on the parameter $s$. 
The integrands $\boldsymbol{p}_{j}(s)=\left[\underline{p}_{j}(s), \bar{p}_{j}(s)\right]$ and $\boldsymbol{u}_{j}(s)=\left[\underline{u}_{j}(s), \bar{u}_{j}(s)\right]$ are the interval parametric boundary functions defined on corresponding boundary segments $S_{j}$ (that theoretically dividing the boundary of the problem). Respectively function $\boldsymbol{u}$ represent the Dirichlet boundary condition when $\boldsymbol{p}$ is the Neumann boundary condition. One of these functions has been defined as uncertain (interval) boundary condition on segment $\mathbf{S}_{j}$, while the other one has been found as a numerical solution of interval PIES (8).

The kernels (integrands) $U_{l j}^{*}$ and $P_{l j}^{*}$ are defined as follow:

$$
U_{l j}^{*}\left(s_{1}, s\right)=\frac{1}{2 \pi} \ln \frac{1}{\left[\eta_{1}^{2}+\eta_{2}^{2}\right]^{0.5}}, \quad P_{l j}^{*}\left(s_{1}, s\right)=\frac{1}{2 \pi} \frac{\eta_{1} n_{1}(s)+\eta_{2} n_{2}(s)}{\eta_{1}^{2}+\eta_{2}^{2}},
$$

where $n_{1}(s)$ and $n_{2}(s)$ are components of the normal vector $n_{j}$ to the boundary segment $\boldsymbol{S}_{j}$ specified by $j$ index. Kernels $U_{l j}^{*}$ and $P_{l j}^{*}$ allow the analytical inclusion of the boundary shape in their mathematical formalism. The shape can be defined by appropriate dependencies between segments $\boldsymbol{S}_{m}(m=l, j=1,2,3, \ldots, n)$ :

$$
\eta_{1}=S_{l}^{(1)}\left(s_{1}\right)-S_{j}^{(1)}(s), \quad \eta_{2}=S_{l}^{(2)}\left(s_{1}\right)-S_{j}^{(2)}(s) .
$$

The segments specified by $\boldsymbol{S}_{m}$ should be defined as closed curve segments.

\section{VERIFICATION OF INTERVAL PIES SOLUTIONS}

The verification of obtained interval PIES solutions is presented based on boundary value problems modeled by Laplace's equation with uncertainly defined boundary conditions. The exactly defined (without uncertainty) PIES method is used for comparison. The examples of constant boundary conditions as well as defined by the linear function are discussed. Additionally, the problems with defined Dirichlet and mixed boundary conditions are presented.

\subsection{Example 1. The uncertainty of constant boundary condition}

The first example is the octagonal domain. The constant Dirichlet (Fig. 1a) and mixed (Fig. 1b) boundary conditions are considered. Interval solutions inside the domain of the so-defined problem, obtained using the interval PIES method, are presented (as the middle of the interval and the half of it width) in Tab. 1. The comparison with solutions obtained using exactly defined (without uncertainty) PIES method has been
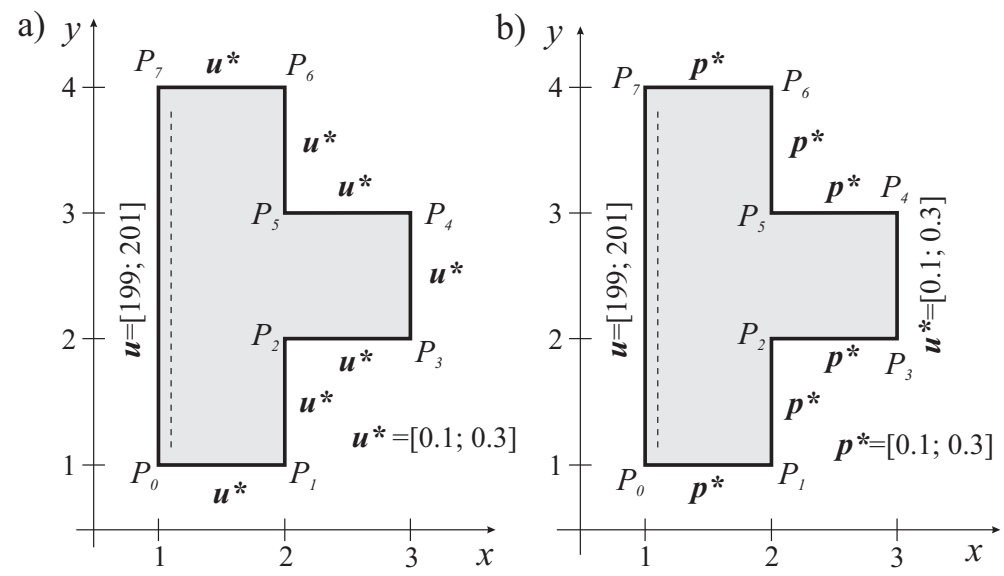

Figure 1. The definition of uncertainly defined constant boundary conditions a) Dirichlet, b) mixed.

proposed for verification. The errors of PIES solutions have been obtained using the total differential method. This method is used to calculate errors of the function. If absolute errors of all function arguments are known as $\left|\Delta x_{i}\right|(i=1,2, \ldots, n)$ and the function $u=f\left(x_{1}, x_{2}, \ldots, x_{n}\right)$ is differentiable, then the absolute error of $u$ function can be defined as follows (Peng et al., 2013):

$$
\Delta u=\sum_{i=0}^{n}\left|\frac{\partial f}{\partial x_{i}}\right|\left|\Delta x_{i}\right|, \quad \frac{\partial f}{\partial x_{i}}=\frac{f\left(x_{i}+h\right)-f\left(x_{i}\right)}{h},
$$


where, in boundary value problems, the function $f$ corresponds to PIES solutions and the arguments of the function are the uncertainly defined boundary c onditions. The function is d efined by nu merical solutions, so the differential can not be obtained analytically. Therefore, to obtain differential from (7), the numerical differential has been used. The value $h=0.00001$ and the $f$ function is defined by PIES s olutions. Correspondingly, the $f\left(x_{i}\right)$ is obtained for middle values of uncertainly defined boundary conditions and $f\left(x_{i}+h\right)$ with a small change of boundary conditions. Such solutions are presented in Tab. 1 as PIES solutions. Satis-

Table 1. Solutions in domain - uncertainly defined boundary conditions.

\begin{tabular}{cccccccccc}
\hline & & \multicolumn{3}{c}{ Dirichlet boundary conditions } & \multicolumn{3}{c}{ mixed boundary conditions } \\
cross-section & \multicolumn{2}{c}{ PIES } & \multicolumn{2}{c}{ interval PIES } & \multicolumn{2}{c}{ PIES } & \multicolumn{3}{c}{ interval PIES } \\
\hline$x$ & $y$ & $u_{p}$ & $\Delta u_{p}$ & mid $(\boldsymbol{u})$ & $\Delta u$ & $u_{p}$ & $\Delta u_{p}$ & mid $(\boldsymbol{u})$ & $\Delta u$ \\
\hline 1.1 & 1.1 & 84.267 & 0.479 & 84.267 & 0.478 & 197.220 & 1.007 & 197.220 & 1.014 \\
1.1 & 1.45 & 163.672 & 0.826 & 163.672 & 0.835 & 194.324 & 0.990 & 194.324 & 0.989 \\
1.1 & 1.8 & 171.902 & 0.869 & 171.902 & 0.871 & 190.257 & 0.962 & 190.257 & 0.965 \\
1.1 & 2.15 & 172.343 & 0.873 & 172.343 & 0.872 & 186.802 & 0.954 & 186.802 & 0.947 \\
1.1 & 2.5 & 203.859 & 1.028 & 203.859 & 1.028 & 215.808 & 1.093 & 215.808 & 1.090 \\
1.1 & 2.85 & 172.343 & 0.873 & 172.343 & 0.872 & 186.802 & 0.954 & 186.802 & 0.947 \\
1.1 & 3.2 & 171.902 & 0.869 & 171.902 & 0.871 & 190.257 & 0.962 & 190.257 & 0.965 \\
1.1 & 3.55 & 163.672 & 0.826 & 163.672 & 0.835 & 194.324 & 0.990 & 194.324 & 0.989 \\
1.1 & 3.9 & 84.267 & 0.479 & 84.267 & 0.478 & 197.220 & 1.007 & 197.220 & 1.014 \\
\hline \multicolumn{3}{c}{ Average relative error [\%] } & 0.00 & 0.35 & & & 0.00 & 0.46 \\
\hline
\end{tabular}

factory solutions have been obtained as a result. The average relative error is below $0.5 \%$, either for Dirichlet boundary conditions and mixed. Therefore, the proposed strategy seems to work properly with uncertainly defined boundary conditions.

Additionally, it was decided to compare interval solutions with the set of solutions obtained using exactly defined PIES. The s et of b oundary value p roblems d efined in side the in tervals (d efining the unc ertainty of boundary conditions) has been considered. The selection of exactly defined problems bases on a combination of lower and upper bounds of boundary conditions. Therefore, the number of all such combinations is exactly $2^{8}=256$ (where 2 - correspond to selection between lower and upper bound, then 8 means the number of segments).

In Tab. 2, the min and max values means the minimum and maximum values from the mentioned set of PIES solutions. Obtained values are the same as the bounds of the interval PIES solutions. Certainly, it should be clearly emphasized, that the computational time of the interval PIES solution is far lower than using 256 times exactly defined PIES method.

Table 2. Solutions in domain - uncertainly defined boundary conditions.

\begin{tabular}{ccccccc}
\hline & \multicolumn{2}{c}{ Dirichlet boundary conditions } & \multicolumn{2}{c}{ mixed boundary conditions } \\
$\begin{array}{c}\text { cross-section } \\
(\mathrm{x}, \mathrm{y})\end{array}$ & interval PIES & \multicolumn{2}{c}{ PIES } & interval PIES & \multicolumn{2}{c}{ PIES } \\
\hline$(1.1,1.1)$ & {$[83.789 ; 84.746]$} & 83.789 & 84.746 & {$[196.206 ; 198.234]$} & 196.206 & 198.234 \\
$(1.1,1.45)$ & {$[162.837 ; 164.507]$} & 162.837 & 164.507 & {$[193.335 ; 195.312]$} & 193.335 & 195.312 \\
$(1.1,1.8)$ & {$[171.031 ; 172.772]$} & 171.031 & 172.772 & {$[189.292 ; 191.222]$} & 189.292 & 191.222 \\
$(1.1,2.15)$ & {$[171.471 ; 173.215]$} & 171.471 & 173.215 & {$[185.855 ; 187.748]$} & 185.855 & 187.748 \\
$(1.1,2.5)$ & {$[202.831 ; 204.887]$} & 202.831 & 204.887 & {$[214.717 ; 216.898]$} & 214.717 & 216.898 \\
$(1.1,2.85)$ & {$[171.471 ; 173.215]$} & 171.471 & 173.215 & {$[185.855 ; 187.748]$} & 185.855 & 187.748 \\
$(1.1,3.2)$ & {$[171.031 ; 172.772]$} & 171.031 & 172.772 & {$[189.292 ; 191.222]$} & 189.292 & 191.222 \\
$(1.1,3.55)$ & {$[162.837 ; 164.507]$} & 162.837 & 164.507 & {$[193.335 ; 195.312]$} & 193.335 & 195.312 \\
$(1.1,3.9)$ & {$[83.789 ; 84.746]$} & 83.789 & 84.746 & {$[196.206 ; 198.234]$} & 196.206 & 198.234 \\
\hline
\end{tabular}




\subsection{Example 2. The uncertainty of boundary condition defined by a linear function}

In the next example, the uncertainty of boundary conditions defined by linear function has been considered. The definition of such an example has been presented in Fig. 2. The dotted line represents the cross-section, where the solutions are obtained.

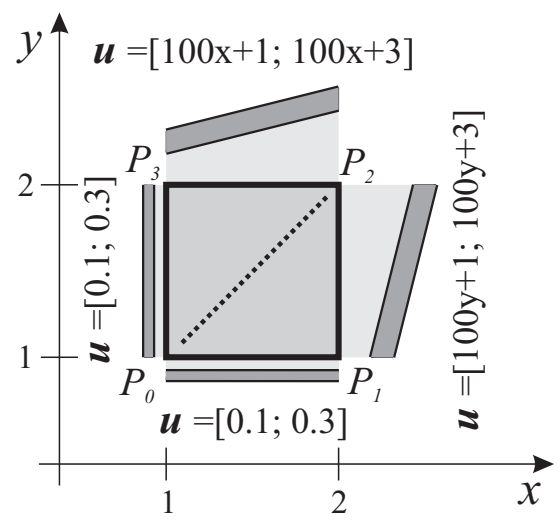

Figure 2. The uncertainty of boundary condition defined by a linear function.

In such an example, the basic square domain Dirichlet problem has been considered. The boundary conditions are defined a s interval c onstant values on two s egments. On the o ther $t$ wo, the conditions a re d efined by interval linear functions. Such functions, to define uncertainty, are shifted by some value (correspondingly down for lower bound and up for upper bound). The exactly defined functions are $100 x+2$ and $100 y+2$.

Interval PIES solutions of so-defined problem ( Fig. 2) a re presented in T ab. 3 . A dditionally, the interval solutions are presented by the middle value of interval and the half of its width. This allows comparing solutions, as in the previous example, with solutions obtained using exactly defined PIES and errors obtained using the total differential method (12). The set of solutions combinations was also considered. Such a set is obtained, analogously as in the previous example, basing on combinations of lower and upper interval bounds (which define boundary condition). The minimum and maximum values of the solutions set are presented by min and max values in Tab. 3 .

Table 3. Solutions in domain - uncertainly defined boundary conditions.

\begin{tabular}{ccccccccc}
\hline \multicolumn{1}{c}{ cross-section } & \multicolumn{3}{c}{ interval PIES } \\
\hline$x$ & $y$ & $\boldsymbol{u}$ & $\operatorname{mid}(\boldsymbol{u})$ & $\Delta u$ & $u_{a}$ & $\Delta u_{a}$ & $\min$ & $\max$ \\
\hline 1.1 & 1.1 & {$[2.86,3.09]$} & 2.978 & 0.116 & 2.978 & 0.116 & 2.86 & 3.09 \\
1.2 & 1.2 & {$[12.81,13.17]$} & 12.990 & 0.178 & 12.990 & 0.177 & 12.81 & 13.17 \\
1.3 & 1.3 & {$[28.69,29.24]$} & 28.961 & 0.275 & 28.961 & 0.275 & 28.69 & 29.24 \\
1.4 & 1.4 & {$[50.07,50.88]$} & 50.473 & 0.403 & 50.473 & 0.402 & 50.07 & 50.88 \\
1.5 & 1.5 & {$[75.55,76.65]$} & 76.100 & 0.550 & 76.100 & 0.550 & 75.55 & 76.65 \\
1.6 & 1.6 & {$[103.03,104.42]$} & 103.727 & 0.697 & 103.727 & 0.692 & 103.03 & 104.42 \\
1.7 & 1.7 & {$[130.41,132.06]$} & 131.239 & 0.825 & 131.239 & 0.840 & 130.41 & 132.06 \\
1.8 & 1.8 & {$[156.29,158.13]$} & 157.210 & 0.922 & 157.210 & 0.908 & 156.29 & 158.13 \\
1.9 & 1.9 & {$[180.24,182.21]$} & 181.228 & 0.984 & 181.228 & 0.982 & 180.24 & 182.21 \\
\hline \multicolumn{3}{c}{ Average relative error [\%] } & & 0.00 & 0.54 & 0.00 & 0.00 \\
\hline
\end{tabular}

Similarly, as in the previous example, the obtained solutions are satisfactory. Middle values of intervals are almost equal to exact solutions. The average relative error, between interval PIES and PIES solutions, is below $0.6 \%$. Additionally, the interval solutions bounds, as in the previous example, are equal to obtained min and max values. 


\section{CONCLUSIONS}

This paper presents the development and verification of the interval PIES method for modeling and solving boundary value problems with uncertainly defined boundary conditions. The special modification of directed interval arithmetic has been also proposed to reduce the solutions overestimation. The proposed interval PIES method has been implemented as a computer program. The verification based on a comparison of interval PIES solutions with solutions obtained using exactly defined PIES method. Obtained interval PIES solutions are satisfactory. Middle values of intervals are almost equal to exact solutions. The average relative error, between interval PIES and PIES solutions for all of the examples, is located between $0-0.54 \%$. Additionally, the interval solutions bounds are equal to the obtained minimum and maximum values of the PIES solutions set. The authors' attention mainly focused on the correctness of IPIES solutions, no on code optimization. The time of the classic PIES method solution is about four times faster than interval PIES. However, even in a basic, four-segment example (to obtain all solutions of the interval boundary conditions combinations), the PIES method should be executed 16 times. So even without optimization, the interval PIES time is much better than the repeated use of classic PURC. Therefore, the obtained results present a high potential of the interval PIES method in modeling and solving uncertainly defined boundary value problems.

\section{REFERENCES}

Spiridonov D., Vasilyeva M., Leung WT. (2019). A Generalized Multiscale Finite Element Method (GMsFEM) for perforated domain flows with Robin boundary conditions, Journal of Computational and Applied Mathematics 357, 319-328.

Sofia A., Romeoc E. (2016). A novel Interval Finite Element Method based on the improvedinterval analysis $311,671-697$.

Kapor J. S., Lucey A. D., Pitman M. W. (2011). A Mesh-Free Compliant-Wall Fluid-Structure Interaction Model, 19th International Congress on Modelling and Simulation, 551-557.

Piasecka-Belkhayat A. (2011). Interval boundary element method for 2D transient diffusion problem using the directed interval arithmetic, Engineering Analysis with Boundary Elements, 35/3, 259-263.

Zieniuk E., Szerszen K. (2009). Triangular Bezier Patches in Modelling Smooth Boundary Surface in exterior Helmholtz Problems Solved by the PIES, Archives of Acoustics 34/1, 51-61.

Kużelewski A., Zieniuk E. (2015). GPU-based acceleration of computations in elasticity problems solving by parametric integral equations system, Advances in Engineering Software 79, 27-35.

Zieniuk E., Kapturczak M. (2018). Modeling the shape of boundary using NURBS curves directly in modified boundary integral equations for Laplace's equation, Computational \& Applied Mathematics 37/4, 48354855 .

Moore R. E. (1966). Interval Analysis. Englewood Cliffs, New York: Prentice-Hall.

Markov S. M. (1992). Extended Interval Arithmetic Involving Infinite Intervals, MathematicaBalkanica, New Series 6, 269-304.

Zieniuk E., Kapturczak M., Kużelewski A. (2016). Solving interval systems of equations obtained during the numerical solution of boundary value problems, Computational \& Applied Mathematics 35/2, 629-638.

Zieniuk E., Kużelewski A., Kapturczak M. (2018). The influence of interval arithmetic on the shape of uncertainly defined domains modelled by closed curves, Computational \& Applied Mathematics 37/2, 10271046.

Peng F.Y., Ma J.Y., Wang W., Duan X.Y., Sun P.P., Yan R. (2013). Total differential methods based universal post processing algorithm considering geometric error for multi-axis NC machine tool, International Journal of Machine Tools \& Manufacture 70, 53-62. 